\title{
Genetic variability, phylogeny and functional implication of the long control region in human papillomavirus type 16, 18 and 58 in Chengdu, China
}

Liyuan Fang ${ }^{1,2}$, Xiaoli Lin ${ }^{1,2}$, Yasi Yang ${ }^{1,2}$, Zhilin Song ${ }^{1,2}$, Xianping Ding ${ }^{1,2^{*}}$ (D) Liping $\operatorname{Tan}^{1,2}$ and Peng Gao ${ }^{1,2}$

\begin{abstract}
Background: Long control region (LCR) of human papillomavirus (HPV) has shown multiple functions on regulating viral transcription. The variations of LCR related to different lineages/sub-lineages have been found to affect viral persistence and cervical cancer progression differently. In this study, we focused on gene polymorphism of HPV16/ 18/58 LCR to assess the effect variations caused on transcription factor binding sites (TFBS) and provided more data for further study of LCR in Southwest China.

Methods: LCR of HPV16/18/58 were amplified and sequenced to do polymorphic and phylogenetic anlysis. Sequences of each type were aligned with the reference sequence by MEGA 6.0 to identify SNPs. Neighbor-joining phylogenetic trees were constructed using MEGA 6.0. Transcription factor binding sites were predicted by JASPAR database.

Results: The prevalence of these three HPVs ranked as HPV16 (12.8\%) > HPV58 (12.6\%) > HPV18 (3.5\%) in Chengdu, Southwest China. 59 SNPs were identified in HPV16-LCR, 18 of them were novel mutations. 30 SNP were found in HPV18-LCR, 8 of them were novel. 55 SNPs were detected in HPV58-LCR, 18 of them were novel. Also, an insertion (CTTGTCAGTTTC) was detected in HPV58-LCR between position 7279 and 7280. As shown in the neighbor-joining phylogenetic trees, most isolates of HPV16/18/58 were clustered into lineage A. In addition, one isolate of HPV16 was classified into lineage $C$ and 3 isolates of HPV58 were classified as lineage B. JASPAR results suggested that TFBS were potentially influenced by $7 / 6$ mutations on LCR of HPV16/18. The insertion and 5 mutations were shown effects in LCR of HPV58.

Conclusion: This study provides more data for understanding the relation among LCR mutations, lineages and carcinogenesis. It also helps performing further study to demonstrate biological function of LCR and find potential marker for diagnosis and therapy.
\end{abstract}

Keywords: Human papillomavirus, Polymorphism, Phylogeny, Transcription factor binding sites prediction

\footnotetext{
* Correspondence: brainding@scu.edu.cn

'Key Laboratory of Bio-Resource and Eco-Environment of Ministry of Education, College of Life Sciences, Sichuan University, Chengdu 610065, Sichuan, People's Republic of China

${ }^{2}$ Bio-resource Research and Utilization Joint Key Laboratory of Sichuan and

Chongqing, Chongqing, People's Republic of China
}

C C The Author(s). 2020 Open Access This article is licensed under a Creative Commons Attribution 4.0 International License, which permits use, sharing, adaptation, distribution and reproduction in any medium or format, as long as you give appropriate credit to the original author(s) and the source, provide a link to the Creative Commons licence, and indicate if changes were made. The images or other third party material in this article are included in the article's Creative Commons licence, unless indicated otherwise in a credit line to the material. If material is not included in the article's Creative Commons licence and your intended use is not permitted by statutory regulation or exceeds the permitted use, you will need to obtain permission directly from the copyright holder. To view a copy of this licence, visit http://creativecommons.org/licenses/by/4.0/ The Creative Commons Public Domain Dedication waiver (http://creativecommons.org/publicdomain/zero/1.0/) applies to the data made available in this article, unless otherwise stated in a credit line to the data. 


\section{Background}

According to the data worldwide in 2012, cervical cancer is the fourth most common cancer in women, both for new cases and deaths. The data show large difference between developed and developing countries. It is the second most common cancer in less developed regions and covered $87 \%$ cervical cancer worldwide, and ranks 11 th in developed regions [1]. There are an estimated 98,900 new cases and 30,500 deaths in China, 2015. That account for 18.7 and $11.5 \%$ of new cervical cancer cases and deaths worldwide, respectively [2].

Human papillomavirus (HPV) is a prevalent, globally distributed group of circular double-stranded DNA virus which can infect cutaneous and mucosal epithelia throughout the human body [3]. Persisting infection of high risk $\mathrm{HPV}$ is the most common reason to develop invasion cervical cancer that is confirmed by the majority. Over 220 HPV types have been fully characterized (https://www. hpvcenter.se/human_reference_clones/). Most oncogenic or high-risk HPV types are members of several species of Alpha-papillomavirus genus $[4,5]$ which are responsible for $90 \%$ of all cervical cancers worldwide [6]. Alphapapillomavirus 7 is mostly related to high-risk mucosal lesion, including HPV18, HPV45, HPV39, etc. Alphapapillomavirus 9 is the most important species to malignant mucosal lesions, including HPV16, HPV33, HPV58, etc. [7]. HPV16/18 are associated with approximately $70 \%$ of invasion cervical cancers worldwide, making them the primary targets for research and vaccination alike [8]. Compelling data demonstrate that HPV16 associated with persistence of infection, development of precancer, progression and histologic type of cervical cancer [9]. The relationship between HPV18 and precancerous lesion is not as compelling as HPV16, but many study have suggested the relevance between HPV18 and cancer [10, 11]. HPV58 has a especially high prevalence in East Asia and ranks the third in cervical cancer cases [12]. Furthermore, it is also frequently appears in precancerous lesions, even more than HPV18, and takes the second slot (overall in $21.1 \%$ of CIN2/3) [13].

The whole genome of HPV contains three regions, an early region (E1, E2, E3, E4, E5, E6, E7), a late region (L1, L2) and a regulatory region called long control region or upstream regulatory region (LCR or URR) [14]. The LCR is an around $850 \mathrm{bp}$ non-coding sequence which has active interaction with many cellular and viral factors. This region include the viral early promoter and transcriptional enhancer, the viral origin of replication, the late polyadenylation site and the late (or negative) regulatory element (LRE/NRE). In this way, it can control late gene expression at various post-transcriptional levels [15].

The LCR has been shown to be the most variable region of HPV genome, mainly because it does not encode any gene and therefore able to accumulate and tolerate more mutations $[16,17]$. The mutations in this region divide HPV into different lineages and sub-lineages which perform differently in viral persistence and progression of precancer/cancer. In HPV16, non-European (sub-lineage A4, B, C, D) variants get three-fold or higher risk to associated with cervical cancer than European (Sub-lineage A1-A3) variants. Non-European variants of HPV18 are also detected more commonly in cancer tissues and high grade cervical lesions [18-20]. HPV58 is the second common HPV-type in Southwest China in previous data $[21,22]$, the variants (C632T and G760A, located on E7) of which have been reported to be highly associated with cervical cancer [23]. The LCR variants have been shown to differently regulate the replication of HPV throughout the viral life cycle [13] and the transcriptional activity of E6 and E7 [14].

In this study, we collected the positive samples of HPV16, 18, 58. Analysis of polymorphism, phylogenetic and functional prediction were performed on LCR which were rarely reported in Chengdu, Southwest China. The data helped us to determine the prevalence of lineages/ sub-lineages and novel mutations/isolates of each type. It is useful for epidemiological survey and biological function research of LCR.

\section{Methods}

\section{Ethical approval and consent to participants}

This study was approved by education and research committee and Ethics Committee of Sichuan University, China (approval number SCU20100196494). All works were followed the guideline of Ethics Committee of Sichuan University. Informed Consent Right was confirmed by patients enrolling. The privacy of patients was assured to protect carefully.

\section{Samples}

All 8244 gynecological outpatients' cervical swab samples were collected between September 2017 and June 2019 in Chengdu SongZiNiao Sterility Hospital, Sichuan Reproductive Health Research Center Affiliated Hospital, Chengdu Western Hospital Maternity Unit, and Angel Women's and Children's Hospital. The samples were collected from women aged 20 to 59 who have normal cytology, low-grade squamous intraepithelial lesion or cervical intraepithelial neoplasia. Each sample was stored at $-20^{\circ} \mathrm{C}$ in cell preservation fluid. Specimen collection to DNA extraction was in a week.

\section{DNA extraction and HPV typing}

DNA was extracted using nucleic acid extraction kit (Health gene technologies, Ningbo, China) in accordance with the manufacturer's instructions. Via capillary electrophoresis method by multiple PCR, DNA extraction was amplified using HPV nucleic acid assay and genotyping kit (Health gene technologies, Ningbo, China). 
Genotyping was accomplished by Applied Biosystems 3500Dx genetic analyzer. The kit can distinguish 25 types (HPV6, 11, 16, 18, 26, 31, 33, 35, 39, 42, 43, 44, 45, $52,53,56,58,59,66,68,73,81,82,83)$ of HPV. HPV $16 / 18 / 58$ positive samples were selected for the following study.

\section{PCR amplification of HPV-LCR}

LCR sequences of HPV16/18/58 were amplified by primers which were designed based on the reference sequences from GenBank (Table 1). PCRs were performed in a final volume of $30 \mu \mathrm{L}$, containing $2 *$ PCR buffer $(200$ $\mathrm{mM}$ Tris- $\mathrm{HCl}$ pH 8.3; $200 \mathrm{mM} \mathrm{KCl}), 2.5$ mM dNTPs, $2 \mathrm{U}$ of EasyTaq DNA Polymerase, and $0.5 \mu \mathrm{M}$ of each primer of the pairs. The PCR program was set as following conditions: an initial denaturation at $95^{\circ} \mathrm{C}$ for $5 \mathrm{~min}$, then entered 30 amplification cycles, $95^{\circ} \mathrm{C}$ for $30 \mathrm{~s}$, primer annealing at $45 \sim 55^{\circ} \mathrm{C}\left(52^{\circ} \mathrm{C}\right.$ for HPV18 and HPV58, $45^{\circ} \mathrm{C}$ for HPV16) for $30 \mathrm{~s}$ and elongation at $72{ }^{\circ} \mathrm{C}$ for $30 \mathrm{~s}$, after $7 \mathrm{~min}$ final extension at $72^{\circ} \mathrm{C}$, ended up and held the temperature at $4{ }^{\circ} \mathrm{C}$. The PCR products were detected using ChemiDoc XRS+ imaging system (Bio-Rad Laboratory, Mississauga, Canada) after electrophoresis through $1.5 \%$ agarose gel. The positive DNA fragments were purified and sequenced by TSINGKE, China.

\section{Analysis of DNA sequences}

To identify the single nucleotide polymorphisms (SNP) in LCR, HPV prototype reference sequences were used as the standard to compare with the valid LCR sequences of each type by MEGA 6.0 [24]. The phylogenetic trees were constructed by the Neighbor-Joining method using Kimura 2Parameter model. The number of bootstrap replications was set at 1000. Sub-lineage reference sequences of the specific type of HPV were participated in constructing the branches of phylogenetic tree (Table 2). All sequences were analyzed using the BLAST (Basic Local Alignment Search Tool) from NCBI (https://blast.ncbi.nlm.nih.gov/Blast.cgi) to detect the novel sites or isolates.

\section{Prediction of transcription factor binding sites}

The online database JASPAR (http://jaspar.genereg.net/) were used to predicted transcription factor binding sites

Table 1 Primer pairs designed for HPV 16/18/58 LCR amplification

\begin{tabular}{llll}
\hline Primer name & Primer sequence & Product size & Anneal \\
\hline HPV-16 R & 5' CACCACCTCATCTACCTCT 3' & $909 \mathrm{bp}$ & $48^{\circ} \mathrm{C}$ \\
HPV-16 F & 5' ACATTGCAGTTCTCTTTTG 3' & & \\
HPV-18 R & 5' TTITGGTTCAGGCTGGATT 3' & $989 \mathrm{bp}$ & $54^{\circ} \mathrm{C}$ \\
HPV-18 F & 5' AGGTAGCTTGTAGGGCTGC 3' & \\
HPV-58 R & 5' CATCCACCAAACGCAAA 3' & $883 \mathrm{bp}$ & $53^{\circ} \mathrm{C}$ \\
HPV-58 F & 5' TCCAACGCCTGACACAA 3' & & \\
\hline
\end{tabular}

(TFBS) in LCR of HPV16/18/58 [25]. Transcription factors that we were interested in were as follows: CEBPB, ETS1, FOS, FOXA1, HSF1, HOXC11,IRF1, IRF2, IRF7, JUN, MAFK, NFIL3, NFKB1, PHOX2A, RAX, SPIB, STAT1, STAT3, SRF, SP1, SRY, SOX9, VAX1 and YY1 for HPV16; CEBPB, ETS1, FOXA1, FOXC1, FOS, GATA3, HOXA10, HOXC11, HOXB13, ID4, JUN, KLF5, NKX6-1, RUNX1, RHOXF1, RAX, SRF, SRY, SOX10, SNAI2, TBR1, TEAD1, VAX1 and YY1 for HPV18; CEBPA, CREB1, ESR2, ETS1, ELK4, FOS, FOXP3, HSF1, HOXC11, IRF2, JUN, NFKB1, NFIA, PHOX2A, POU2F2, RAX, SPIB, SMAD3, STAT1, SOX9, SOX10, SRY, VAX1 and YY1,for HPV58. The relative profile score threshold was set at $85 \%$.

\section{Results}

Characteristics of HPV prevalence in Southwest China

Total 8244 samples were collected from September 2017 to June 2019, 2367 (28.7\%) of them were HPV-positive. Among them, 302(12.8\%), 82(3.5\%) and 299(12.6\%) were tested as HPV16, HPV18 and HPV58, respectively (Fig. 1). The identical sequence was regarded as a pattern. In HPV18, 16 patterns were confirmed from 34 sequences. Pattern No.2 (12 isolates, 35.3\%) was the most prevalence pattern (Table 3). In 57 HPV16 LCR sequences, pattern No.6 (6 isolates, $10.2 \%$ ) was the most common pattern from the 22 patterns (Table 4). Total 31 patterns were identified in 66 HPV58 samples. Pattern No.5 (16 isolates, 24.2\%) was completely same with HPV58 reference sequence which was also the most common pattern (Table 5).

\section{Genomic polymorphisms of HPV-LCR}

30 SNPs were identified in HPV18-LCR. T7592C was found in all isolates. T7258A, C7529A, A7567C and A7670T was the second common mutations in HPV18LCR, got the frequency of $17.6 \%$. These four mutations also appeared together. No insertion or deletion was found. After Blast on NCBI, 8 unique mutations and 9 novel variants were confirmed. (Table 3 ).

59 SNPs were detected in 56 nucleotide positions of HPV16 LCR. The most common mutations, G7193T and G7521A was found in all isolates except HPV16 NC45. A7730C and G7842A were identified in 57.9\% of the variants (33/57). A7175C, T7177C, T7201C and $\mathrm{C} 7270 \mathrm{~T}$ were detected in 32 isolates. No insertion or deletion mutation was found. After Blast on NCBI, we found that 18 mutations and 9 variants were never reported by anyone else. (Table 4).

In HPV58, 55 SNPS were found at 52 nucleotide positions. An insertion (CTTGTCAGTTTC) was detected between the nucleotide sites 7279 and 7280 . The most variable site was 7714 (25/67). All four kinds of nucleotide were found on this position, including 8 of A7714C, 15 of A7714G and 2 of A7714T. The second most prevalence 
Table 2 Reference sequences of LCR

\begin{tabular}{|c|c|c|c|c|c|c|c|c|c|c|}
\hline \multirow{2}{*}{$\frac{\text { HPV type }}{\text { HPV16 }}$} & \multicolumn{10}{|c|}{ Sub-lineages Genbank accession No. } \\
\hline & A1 & A2 & A3 & A4 & B1 & B2 & $\mathrm{C}$ & D1 & D2 & D3 \\
\hline & K027189 & AF536179 & HQ644236 & AF534061 & AF536180 & HQ644298 & AF472509 & HQ644257 & HQ644270 & AF402678 \\
\hline \multirow[t]{2}{*}{ HPV18 } & A1 & A2 & A3 & A4 & A5 & B1 & B2 & B3 & $\mathrm{C}$ & \\
\hline & AY262282 & EF202146 & EF202147 & EF202151 & GQ180787 & EF202155 & KC470225 & EF202152 & KC470229 & \\
\hline \multirow[t]{2}{*}{ HPV58 } & $\mathrm{A} 1$ & A2 & A3 & B1 & B2 & $\mathrm{C}$ & D1 & D2 & & \\
\hline & D90400 & HQ537752 & HQ537758 & HQ537762 & HQ537764 & HQ537774 & HQ537768 & HQ537770 & & \\
\hline
\end{tabular}

mutation was C52T (19/67). Two groups of mutations had a tendency to show together, which were C7265G (12/67), A7714G (15/67) and C7266T (16/67); G7194C (8/57), A7304G (8/57), the insertion (8/57) and A7714C (8/67). 18 novel sites were found in HPV58 which composed 12 novel sequences. (Table 5).

\section{Phylogenetic analysis}

The neighbor-joining phylogenetic trees were constructed by MEGA, using the patterns and reference sequences of sub-lineages .

31 patterns and 9 sub-lineages reference sequences participated in building the tree of HPV16. The phylogenetic tree showed that all patterns were clustered in lineage A,except NC43 (lineage C). The A branch contained 10 patterns (17 isolates) of sub-lineage A1, 4 patterns (7 isolates) of sub-lineage A3 and 18 patterns (32 isolates) of sub-lineage A4. None of patterns belonged to sub-lineage A2 (Fig. 2).

The tree of HPV18 was composed of 16 patterns and 9 sub-lineages reference sequences. All patterns were identified as Lineage A, of which 8 patterns (24 isolates) were divided into sub-lineage A1, 4 patterns (4 isolates) were classified as sub-lineage A2, 1 pattern was identified as sublineage A3 (3 isolates), A4 (1 isolates), respectively. Two patterns (pattern No.34 and No.33) were not clearly identified, while No.33 was more like sub-lineage A5, No.34 was more close to sub-lineage A3 in the tree (Fig. 3).

39 patterns and 7 sub-lineages reference sequences were selected to build the tree of HPV58. All patterns were gathered in lineage $\mathrm{A}$ and $\mathrm{B}$. Lineage $\mathrm{A}$ was the most prevalence sub-type of HPV58, including 19 patterns (38 samples) of sub-lineage A1, 10 patterns (16 samples) of sub-lineage A2 and 8 patterns (9 samples) of sub-lineage A3. Three patterns were classified as lineage B, 2 for Sub-B1 and 1 for sub-B2 (Fig. 4).

\section{Prediction of transcription factor binding sites}

JASPAR database was used to investigate the potential binding sites for the transcription factors in the HPVLCR. Also, it was applied to assess wherther the mutations affected the transcription factor binding sites.

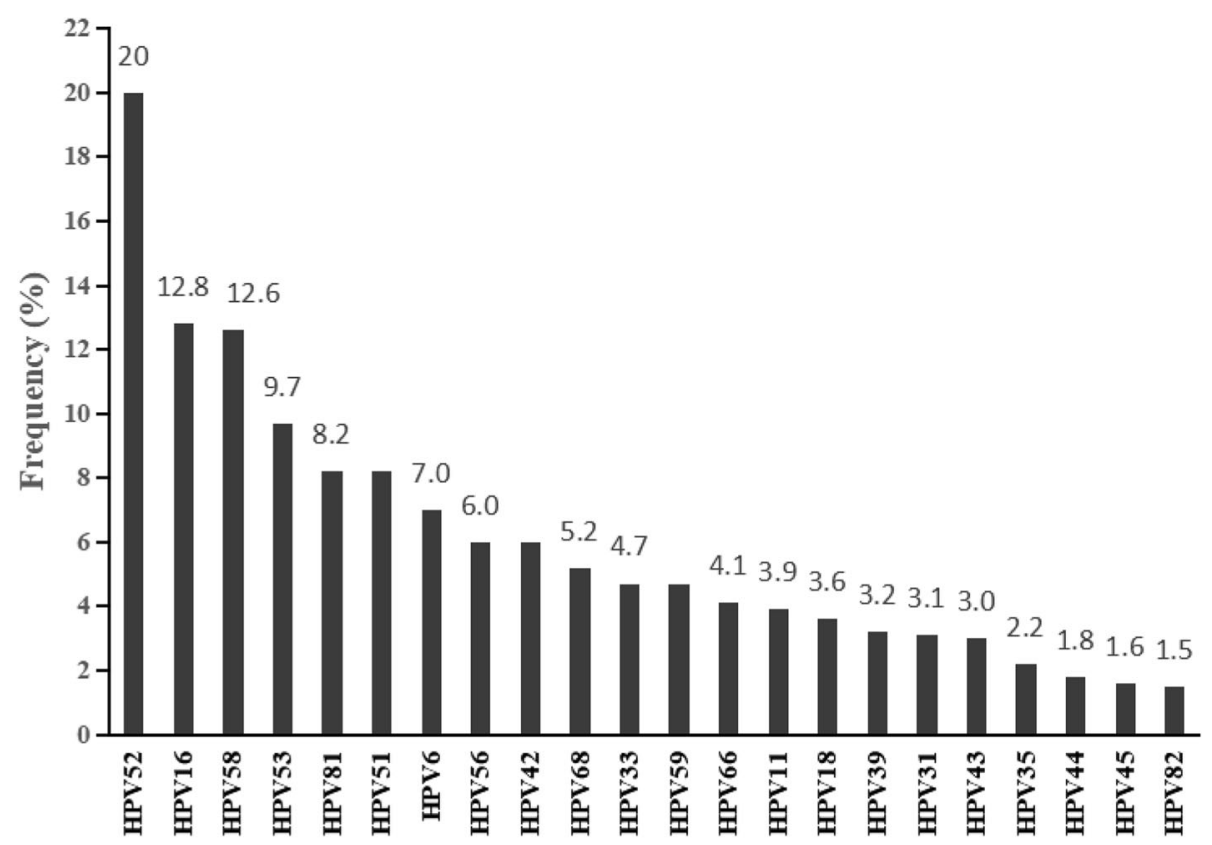

Fig. 1 Prevalence of HPV in Chengdu, Sichuan 


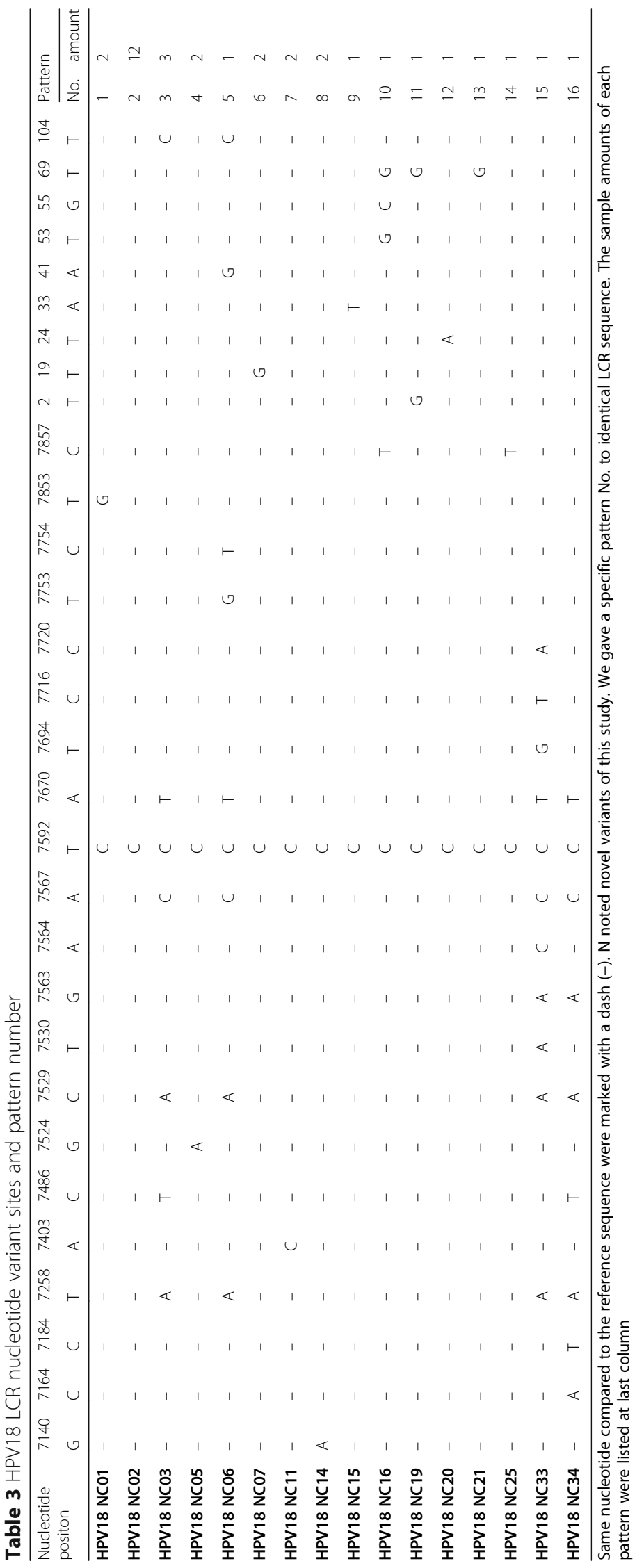




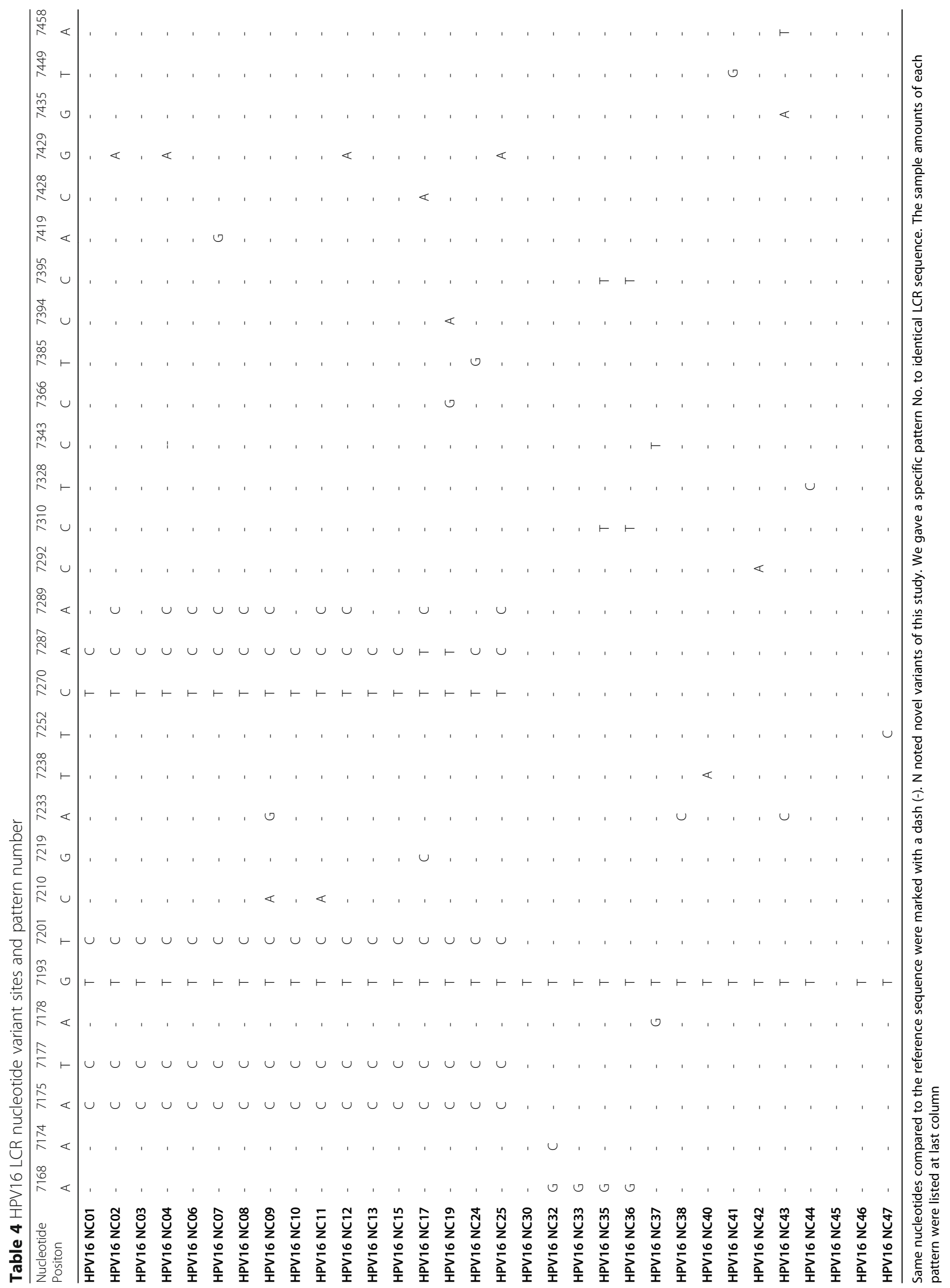




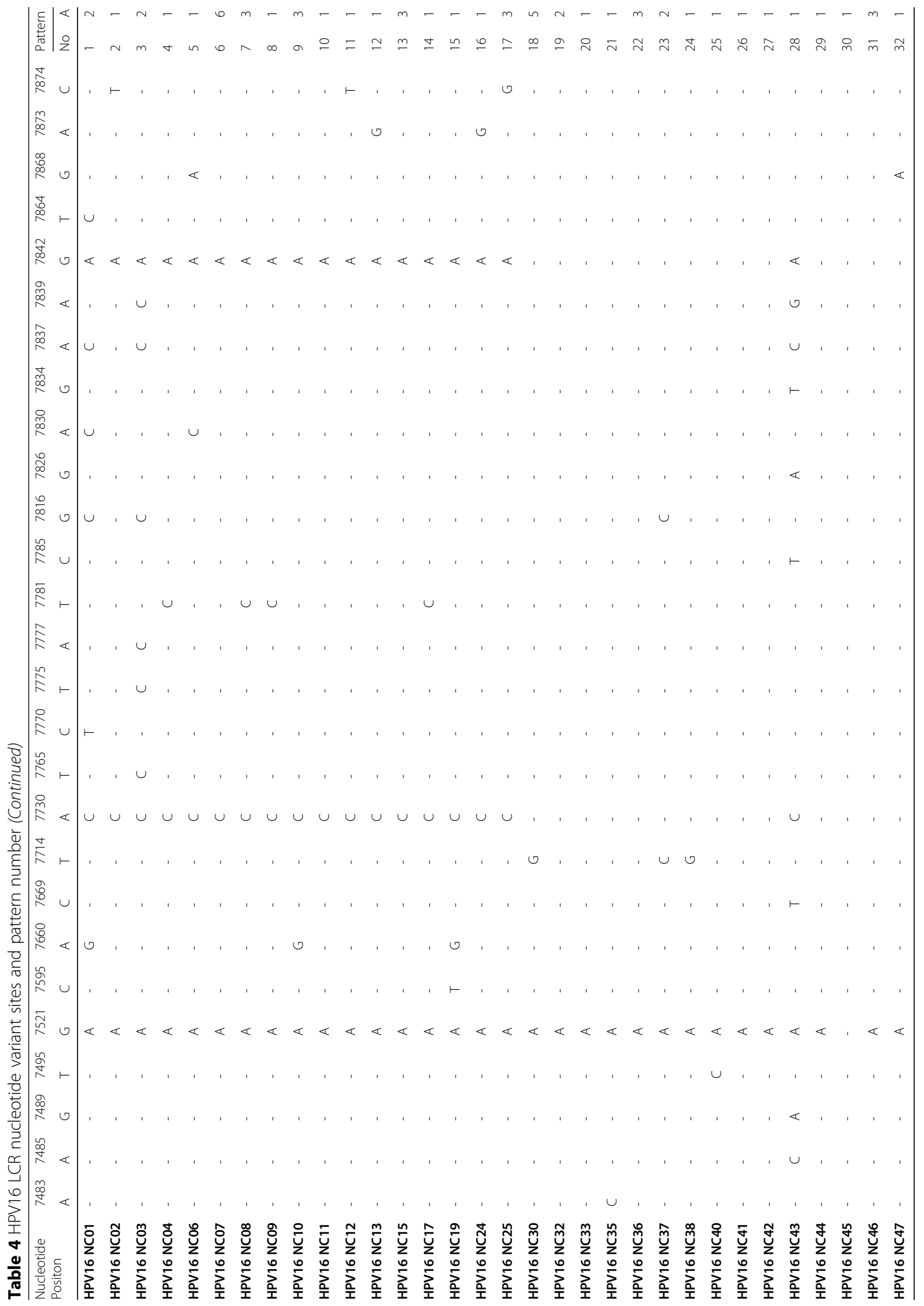









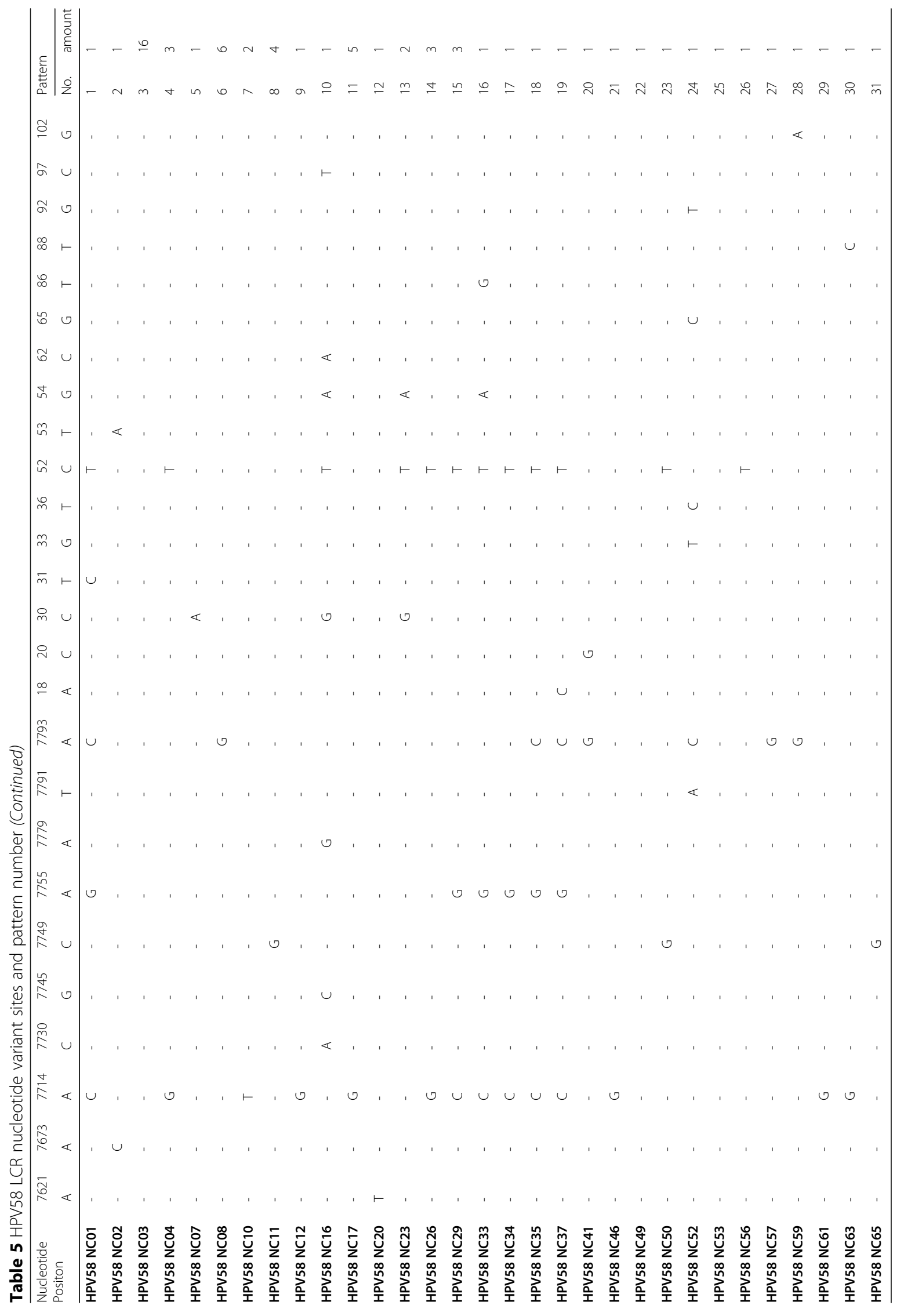






Fig. 2 Neighbor-joining phylogenetic tree of HPV-16 patterns based on LCR sequences. Reference sequences of sub-lineages were marked with red dot 


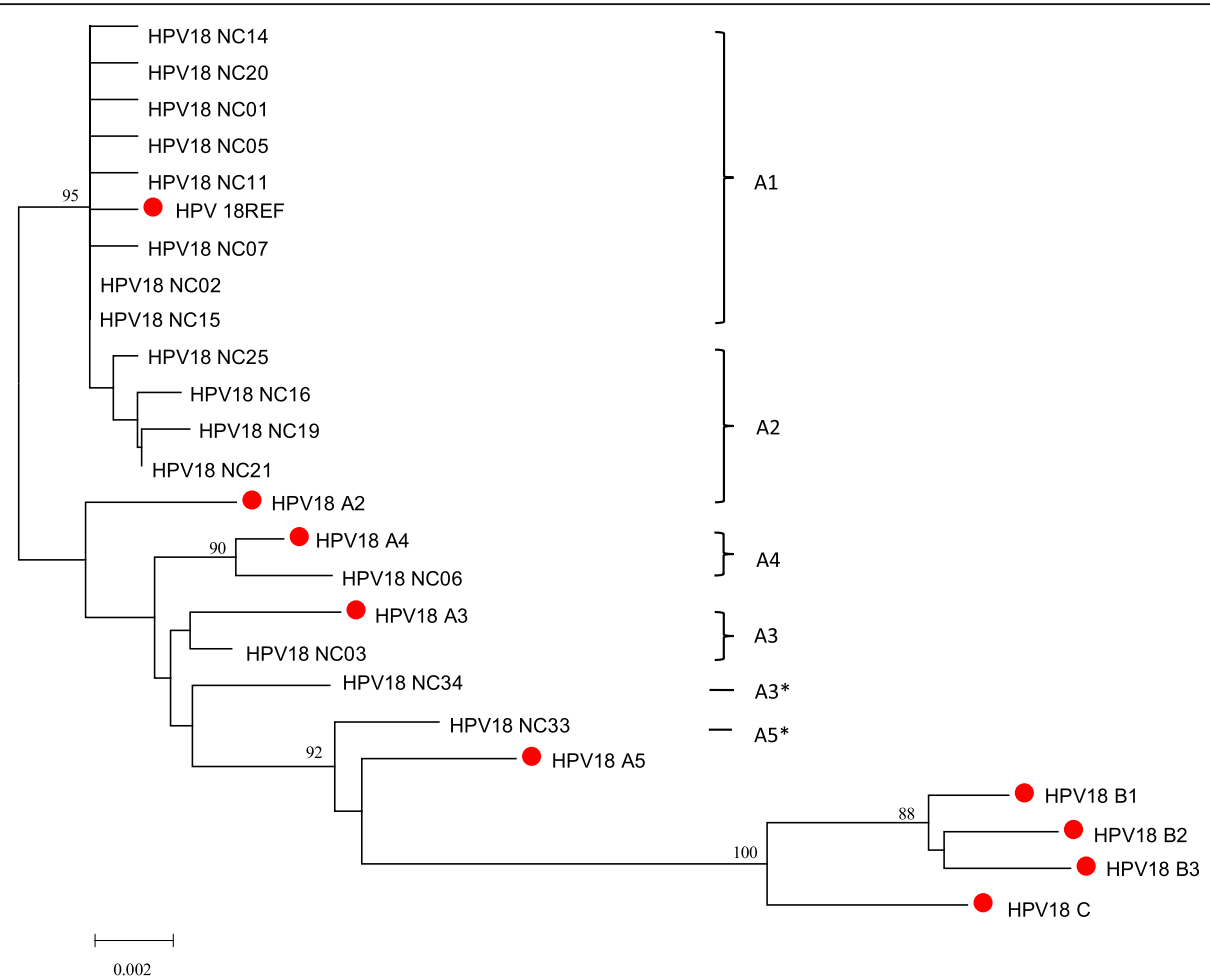

Fig. 3 Neighbor-joining phylogenetic tree of HPV-18 patterns based on LCR sequences. Reference sequences of sub-lineages were marked with red dot; ${ }^{*}$, marked the sub-lineage that the pattern not clearly identified to, but more close to

For HPV16, the results showed 7 mutations potentially affected the binding sites of transcription factors. A potential binding site of PHOX2A located at nucleotide position 7730 which was detected as the second most variable site of HPV16-LCR. More transcription factors like HOXC11, CEBPB, FOS, ETS1 and YY1 were detected on position 7219, 7310, 7366, 7394/7395 and 7595, respectively (Fig. 5a).

The JASPAR result of HPV18-LCR indicated that 6 variations had potential effects on the TFBS. T7592C was detected in all isolates of HPV18 and might be the binding site of GATA3 and SRF. CEBPB was related to variations C7716T and C7720A. C7716T was also potentially affected the binding site of TEAD1. In addition, nucleotide sites 7857,33 and 41 potentially affected the binding sites for HOXA5, FOXL1 and FOXC1, respectively (Fig. 5b).

In HPV58-LCR, 5 variations and 1 insertion were found potential effect on TFBS. A sequence of CTTGTC AGTTTC was inserted in the potential binding sites of transcription factor ETS1 between position 7279 and 7280. More TFs like SOX10, SOX9, CEBPA and ESR2 indicated underlying binding change on account of the mutation C7265G, A7304G, A7376G and T7791A/ A7793C, respectively (Fig. 5c).

\section{Discussion}

Persistence infection of high-risk HPV shows significant link with cervical intraepithelial neoplasia (CIN) and invasion cervical cancer. HPV variants may have codiversified with human populations and thus obtain an intrinsic geographical difference on prevalence and infections [26]. The knowledge of epidemicity about HR-HPV is vital for further study of vaccine and cervical cancer therapy in local area.

These three types of HR-HPV showed different prevalence and mutation rates in our study. More HPV16 and HPV58 were detected than HPV18 in this study. Only one HPV16 isolate was completely same with HPV16 REF sequence, while 16 (24.2\%) isolates were same with REF sequence of HPV58. It may attributed to the high prevalence and active interaction in viral genome integration and cancer development of HPV16, so the LCR were able to occur and reserve more variations. HPV18 is less popular than HPV16/58 in Southwest China, and we didn't find as many mutations as those two types in 18. T7592C was a general mutation for HPV18 in this area. It was found in all isolates of HPV18 and also the only variation of $35.3 \%$ (12 isolates) HPV18 LCR sequence. HPV58 showed different prevalence through geographical regions. The unusual high prevalence had been reported in East Asia, Africa and some other areas [27]. A few study reported the high prevalence of HPV58 in Japan (8\%) and Korea (16\%) [28-30]. About the data in China, infection frequency of HPV58 was found 9.4\% in Zhejiang [31], 10\% in Hong Kong [32] 


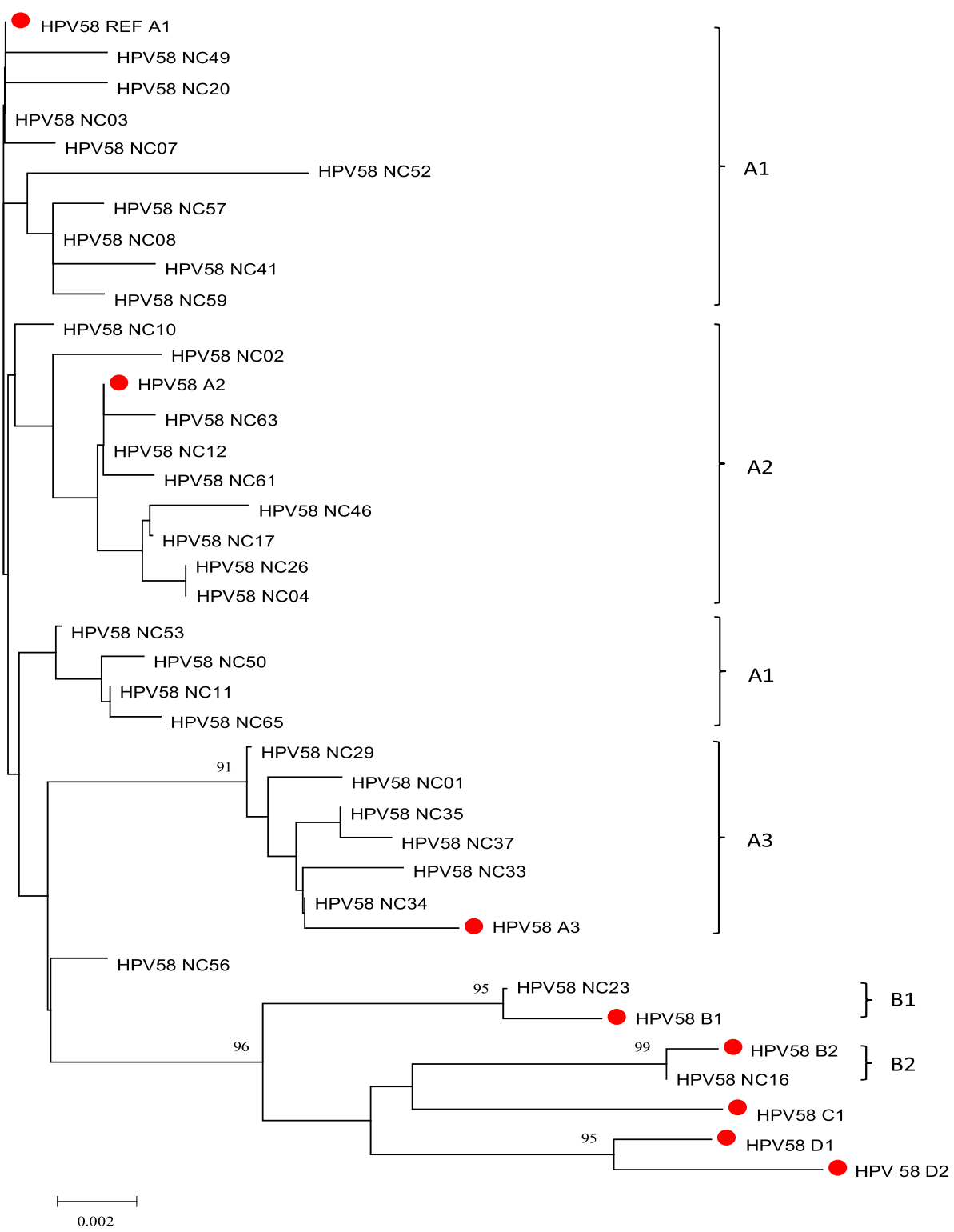

Fig. 4 Neighbor-joining phylogenetic tree of HPV-58 patterns based on LCR sequence. Reference sequences of sub-lineages were marked with red dot

and $10 \%$ in Taiwan [33]. In this study, HPV58 occupied $12 \%$ of the positive samples. It suggested that further study of vaccine need to consider this type as target.

The distribution of the sub-lineages were basically same with the data had been reported previously. In this study, A lineage accounted for a large proportion of all three types. It has been reported that lineage A related to pathogenicity more than other lineages.

For HPV16, a worldwide phylogenetic analysis indicated that the EUR lineages $(\mathrm{A} 1 \sim \mathrm{A} 3)$ were epidemic in many regions. A4 was largely found in Eastern Asia [34]. $56 \%$ of HPV16 variants were clustered into sub-lineage
A4 which was significantly associated with elevated risk of cervical cancer comparing with A1 3 [35].

All HPV18 isolated were identified as lineage A. The global data of lineage A showed that sub-lineage A1 predominated in Eastern Asia and Pacific, while sub-lineage A3/A4 were prevalent in many region around the world. The isolates of A5 were detected principally in Africa [36]. Our data showed that HPV18 samples were mostly identified as A1 and a small amount of A2/A3/A4. $70.6 \%$ isolates of HPV18 were clustered in sub-lineage A1. Amador-Molina et al. found that the variations of sub-A1 affected Ori function significant higher than the 


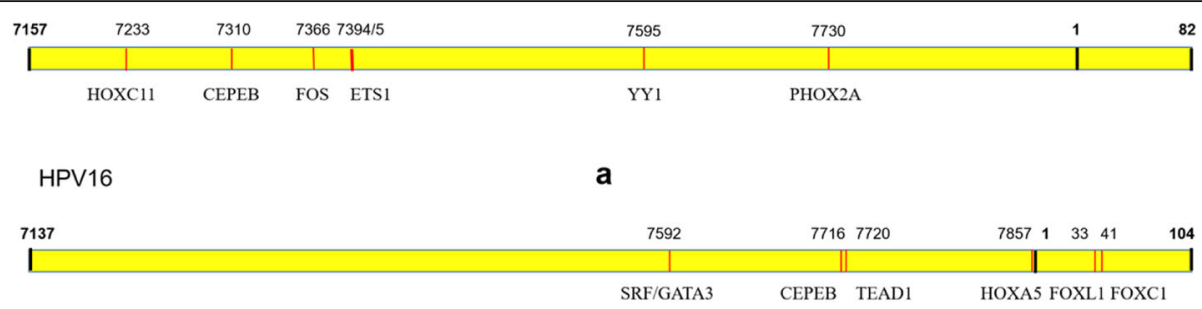

HPV18

b

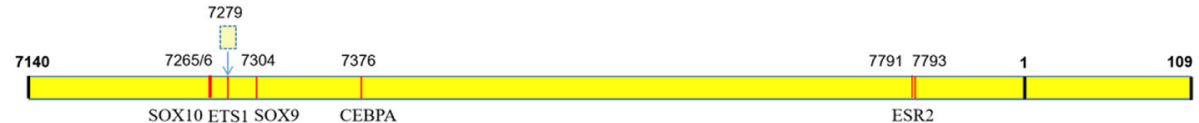

HPV58

C

Fig. 5 Transcription factors prediction on LCR. (a): transcription factors prediction of HPV16. (b): transcription factors prediction of HPV18. (c): transcription factors prediction of HPV58. The JASPAR database was used to investigate the potential binding sites within HPV-LCR. The yellow strips represent sequence of LCR.: Bold black line marked boundary positions of LCR and initiation sites of genome.: Red line marked variants sites which may effect binding sites of transcription factors.: The block with dash line present the insertion we found in HPV58

other variants [37]. This effect may be related to the changes found in the keratinocyte enhancer (KE) region of LCR as it has been reported that mutations in this domain affect HPV replication [38].

For HPV58, globally, the sub-lineage A2 was the most widespread variant, whereas sub-lineages A1 and A3 were rarely found out of Asia. A1 were the most prevalence sub-lineage of HPV58 in our study, A2/A3 were also be found. $95.4 \%$ of the HPV58 isolates were clustered in lineage $\mathrm{A}$ which showed much more relation with CIN2/CIN3+ rather than lineage BCD [30]. We also found 3 variants of lineage $B$ which was rarely observed in East Asia [39].

The HPV LCR which contain the binding sites for both viral and cellular factors, has shown regulatory functions on replication of HPV, transcriptional activity of the E6/E7 and the other interaction through the virus life cycle $[10,13,28]$. Mutations on LCR may influence the binding sites and the function of it. The mutations of some sub-lineages which are more related to pathgenicity, showed potential effect on TFBS in our data. In HPV16, A7730C, variant of sub-lineage A4, showed potentially effect to PHOX2A which is a transcription factor involving in cell proliferation and migration in lung cancer [40]. CEBPB, FOXL1 and HOXA5 were the transcription factors that may be affected in sub-lineage A1 of HPV18. CEBPB is a leucine-zipper transcription factor that regulates growth and differentiation of hematopoietic and epithelial cells. One study based on breast cancer found that $\mathrm{CEBPB}$ was a novel transcriptional regulator of CLDN4. The upregulation of CEBPBCLDN4 signaling caused the migration and invasion of cancer cell [41]. Homeobox A5 (HOXA5) is a member of the homeobox (HOX) family and is upregulated in many types of tumors [42]. Forkhead box L1 (FOXL1) is a member of the Forkhead box (FOX) superfamily and was reported to be dysregulated in various types of cancers. Upregulation of FOXL1 greatly inhibits cell proliferation, migration, and invasion in vitro and tumorigenicity in nude mice [43]. In HPV58, the nucleotide sites 7265 and 7266 were not only the most variable sites in LCR but also the potential binding sites of SOX10 which is a transcription factor of sex determining region Y (SRY)-related high motility group (HMG)box gene family. SOX10 has been suggested as a useful marker for corresponding tumors [44], although it was usually silenced or downregulated in malignant tumors such as digestive cancers [45] and prostatic carcinoma [46]. The insertion (between 7279 and 7280) which is usually detected in sub-lineage A3, may affect the binding site of ETS1. ETS1 belongs to the large family of the ETS domain family of transcription factors and is involved in cancer progression. Mostly, ETS1 expression is linked to poor survival and contributed to the acquisition of cancer cell invasiveness, EMT (epithelial-to-mesenchymal transition), the development of drug resistance and neo-angiogenesis [47].

\section{Conclusion}

In conclusion, this study investigated the gene polymorphisms, phylogeny, and relevant functional prediction of high-risk HPV-LCR from Southwest China. Although our study showed some limitations on sample capacity and source, it provided more data for understanding the intrinsic geographical relatedness of HPV-16/18/58 variants, the complicated relation among HPV-16/18/58 LCR mutations, transcription factors and carcinogenesis. It also helps performing further study to demonstrate 
the biological function of HPV-16/18/58 LCR variants and the effect of multiple infection of high-risk HPV on tumor progression. The TFBS we found is still need deeper exploration for the potential of them to be marker in diagnosis and therapy.

\section{Abbreviations}

DNA: Deoxyribonucleic Acid; HPV: Human Papillomavirus; LCR: Long control region; MEGA: Molecular Evolutionary Genetics Analysis; NCBI: National Center for Biotechnology Information; PCR: Polymerase Chain Reaction; SNP: Single nucleotide polymorphism; TFBS: transcription factor binding site

\section{Acknowledgements}

This work was funded by Key Scientific Research Foundation Projects of Sichuan Province and supported by following hospitals: The Angel Women's and Children's Hospital, The Sichuan Reproductive Health Research Center Affiliated Hospital, The Chengdu Western Hospital Maternity Unit, Chengdu Songziniao Sterility Hospital and others that participated in this study.

\section{Ethics approval and consent to participants}

This study was approved by education and research committee and Ethics Committee of Sichuan University, China (approval number SCU20100196494). All works were followed the guideline of Ethics Committee of Sichuan University. Informed Consent Right was confirmed by patients enrolling. The privacy of patients was assured to be protected carefully.

\section{Authors' contributions}

Conception of the study and supervising the progress of study: XD and LF. Conducting the experiments.: LF. sample collection: LF and ZS. HPV typing: $L F, Z S, X L, L T$ and $P G$. Other parts of experiments: LF, $X L$. Data analysis: LF and $Y Y$. Draft of manuscript: LF. All authors reviewed the manuscript. All authors read and approved the final manuscript.

\section{Funding}

The present study was funded by Key Scientific Research Foundation

Projects of Sichuan Province (No. 2018JY0601).

\section{Availability of data and materials}

All data generated or analyzed during this study are included in this article and Genbank.

\section{Consent for publication}

Not applicable.

\section{Competing interests}

The authors declare that they have no competing interests.

Received: 6 November 2019 Accepted: 3 June 2020

Published online: 16 July 2020

\section{References}

1. Ferlay J, Soerjomataram I, Dikshit R, Eser S, Mathers C, Rebelo M, et al. Cancer incidence and mortality worldwide: sources, methods and major patterns in GLOBOCAN 2012. Int J Cancer. 2015;43:66-7.

2. Chen W, Zheng R, Baade PD, Zhang S, Zeng H, Bray F, et al. Cancer statistics in China, 2015. CA Cancer J Clin. 2016;66:115-32.

3. Burk RD, Harari A, Chen Z. Human papillomavirus genome variants. Virology. 2013:445:232-43.

4. Ghittoni R, Accardi R, Chiocca S, Tommasino M. Role of human papillomaviruses in carcinogenesis. Ecancermedicalscience. 2015;9:1-9.

5. Muñoz N, Bosch FX, De Sanjosé S, Herrero R, Castellsagué X, Shah KV, et al. Epidemiologic classification of human papillomavirus types associated with cervical cancer. N Engl J Med. 2003;348:518-27.

6. Schiffman M, Herrero R, Desalle R, Hildesheim A, Wacholder S, Rodriguez AC, et al. The carcinogenicity of human papillomavirus types reflects viral evolution. Virology. 2005;337:76-84.

7. de Villiers E, Fauquet C, Broker TR, Bernard H,zur Hausen H. Classification of Papillomaviruses Virology2004;324:17-27.

8. Li N, Franceschi S, Howell-Jones R, Snijders PJF, Clifford GM. Human papillomavirus type distribution in 30,848 invasive cervical cancers worldwide: variation by geographical region, histological type and year of publication. Int J Cancer. 2011;128:927-35.

9. Hildesheim A, Schiffman M, Bromley C, Herrero R, Rodriguez C, Bratti C, et al. Human papillomavirus type 16 variants and risk of cervical cancer. J Natl Cancer. 2001;93:315-8.

10. zur Hausen H. Papillomaviruses and cancer: from basic studies to clinical application. Nat Rev Cancer. 2002;2:342-350.

11. Wu Z, Qin Y, Yu L, Lin C, Wang H, Cui J, et al. Association between human papillomavirus HPV16, HPV18, and other HR-HPV viral load and the histological classification of cervical lesions: results from a large-scale crosssectional study. J Med Virol. 2017;89:535-41.

12. Bao YP, Li N, Smith JS, Qiao YL, Tan J, Liu J, et al. Human papillomavirus type distribution in women from Asia: a meta-analysis. Int J Gynecol Cancer. 2008;18:71-9.

13. Guan P, Howell-Jones R, Li N, Bruni L, De Sanjosé S, Franceschi S, et al. Human papillomavirus types in 115,789 HPV-positive women: a metaanalysis from cervical infection to cancer. Int J Cancer. 2012;131:2349-59.

14. Burk RD, Chen Z, Van Doorslaer K. Human papillomaviruses: genetic basis of carcinogenicity. Public Health Genomics. 2009;12:281-90.

15. Graham SV. Keratinocyte differentiation-dependent human papillomavirus gene regulation. Viruses. 2017;9:245-62.

16. Ho L, Chan SY, Chow V, Chong T, Tay SK, Villa LL, et al. Sequence variants of human papillomavirus type 16 in clinical samples permit verification and extension of epidemiological studies and construction of a phylogenetic tree. J Clin Microbiol. 1991;29:1765-72.

17. Yamada T, Manos MM, Peto J, Greer CE, Munoz N, Bosch FX, et al. Human papillomavirus type 16 sequence variation in cervical cancers: a worldwide perspective. J Virol. 1997;71:2463-72.

18. Cornet I, Gheit T, lannacone MR, Vignat J, Sylla BS, Del Mistro A, et al. HPV16 genetic variation and the development of cervical cancer worldwide. $\mathrm{Br} \mathrm{J}$ Cancer. 2013;108:240-4.

19. de Sanjose S, Quint WGV, Alemany L, Geraets DT, Klaustermeier JE, Lloveras $B$, et al. Human papillomavirus genotype attribution in invasive cervical cancer: a retrospective cross-sectional worldwide study. Lancet Oncol. 2010; 11:1048-56.

20. Xi LF, Kiviat NB, Hildesheim A, Galloway DA, Wheeler CM, Ho J, et al. Human papillomavirus type 16 and 18 variants: race-related distribution and persistence. J Natl Cancer Inst. 2006;98:1045-52.

21. Xi J, Chen J, Xu M, Yang H, Wen S, Pan Y, et al. The polymorphisms of LCR, E6, and E7 of HPV-58 isolates in Yunnan. Southwest China Virol J. 2018;15:76.

22. Li J, Mei J, Wang X, Hu L, Lin Y, Yang P. Human papillomavirus type-specific prevalence in women with cervical intraepithelial neoplasm in Western China. J Clin Microbiol. 2012;50:1079-81.

23. Chan PK, Lam CW, Cheung TH, Li WW, Lo KW, Chan YM, et al. (2002). Association of Human Papillomavirus Type 58 variant with the risk of cervical Cancer. CancerSpectrum Knowl Environ. 2002;94:1249-53.

24. Tamura K, Stecher G, Peterson D, Filipski A, Kumar S. MEGA6: molecular evolutionary genetics analysis version 6.0. Mol Biol Evol. 2013;30:2725-9.

25. Mathelier A, Fornes O, Arenillas DJ, Chen CY, Denay G, Lee J, et al. JASPAR 2016: a major expansion and update of the open-access database of transcription factor binding profiles. Nucleic Acids Res. 2016;44:D110-5.

26. Chen Z, Schiffman M, Herrero R, DeSalle R, Anastos K, Segondy M, et al. Evolution and taxonomic classification of human papillomavirus 16 (HPV16)related variant genomes: HPV31, HPV33, HPV35, HPV52, HPV58 and HPV67. PLoS One. 2011;6.

27. Clifford GM, Smith JS, Plummer M, Muñoz N, Franceschi S. Human papillomavirus types in invasive cervical cancer worldwide: a meta-analysis. Br J Cancer. 2003;88:63-9.

28. Hwang T. Detection and typing of human papillomavirus DNA by PCR using consensus primers in various cervical lesions of Korean women. J. Korean Med. Sci. 1999. p. 593-9.

29. Onuki M, Matsumoto K, Satoh T, Oki A, Okada S, Minaguchi T, et al. Human papillomavirus infections among Japanese women: age-related prevalence and type-specific risk for cervical cancer. Cancer Sci. 2009;100:1312-6.

30. Azuma Y, Kusumoto-Matsuo R, Takeuchi F, Uenoyama A, Kondo K, Tsunoda $\mathrm{H}$, et al. Human papillomavirus genotype distribution in cervical intraepithelial neoplasia grade 2/3 and invasive cervical cancer in Japanese women. Jpn J Clin Oncol. 2014;44:910-7.

31. Hong D, Ye F, Chen H, Lü W, Cheng Q, Hu Y, et al. Distribution of human papillomavirus genotypes in the patients with cervical carcinoma and its precursors in Zhejiang Province. China Int J Gynecol Cancer. 2008;18:104-9. 
32. Chan PKS, Ho WCS, Yu MN, Pong WM, Chan ACL, Chan AKC, et al. Distribution of human papillomavirus types in cervical cancers in Hong Kong: current situation and changes over the last decades. Int J Cancer. 2009:125:1671-7.

33. Ding DC, Hsu HC, Huang RL, Lai HC, Lin CY, Yu MH, et al. Type-specific distribution of HPV along the full spectrum of cervical carcinogenesis in Taiwan: an indication of viral oncogenic potential. Eur J Obstet Gynecol Reprod Biol. 2008;140:245-51.

34. Cornet I, Gheit T, Franceschi S, Vignat J, Burk RD, Sylla BS, et al. Human papillomavirus type 16 genetic variants: phylogeny and classification based on E6 and LCR. J Virol. 2012;86:6855-61.

35. Hang D, Yin Y, Han J, Jiang J, Ma H, Xie S, et al. Analysis of human papillomavirus 16 variants and risk for cervical cancer in Chinese population. Virology. 2016:488:156-61.

36. Chen AA, Gheit T, Franceschi S, Tommasino M, Clifford GM. Human papillomavirus 18 genetic variation and cervical Cancer risk worldwide. J Virol. 2015;89:10680-7.

37. Amador-Molina A, González-Montoya JL, García-Carrancá A, Mohar A, Lizano M. Intratypic changes of the E1 gene and the long control region affect ori function of human papillomavirus type 18 variants. J Gen Virol. 2013;94:393-402.

38. Hubert WG, Kanaya T, Laimins LA. DNA replication of human papillomavirus type 31 is modulated by elements of the upstream regulatory region that lie 5' of the minimal origin. J Virol. 1999;73:1835-45.

39. Chen Z, Ho WCS, Boon SS, Law PTY, Chan MCW, DeSalle R, et al. Ancient evolution and dispersion of human papillomavirus 58 variants. J Virol. 2017;91.

40. Wang R, Chen X, Xu T, Xia R, Han L, Chen W, et al. MiR-326 regulates cell proliferation and migration in lung cancer by targeting phox $2 a$ and is regulated by HOTAIR. Am J Cancer Res. 2016;6:173-86.

41. Wang F, Gao Y, Tang L, Ning K, Geng N, Zhang H, et al. A novel PAK4CEBPB-CLDN4 axis involving in breast cancer cell migration and invasion. Biochem Biophys Res Commun. 2019;511:404-8.

42. Zhang H, Zhao J, Suo Z. Knockdown of HOXA5 inhibits the tumorigenesis in esophageal squamous cell cancer. Biomed Pharmacother. 2017;86:149-54.

43. Qin Y, Gong W, Zhang M, Wang J, Tang Z, Quan Z. Forkhead box L1 is frequently downregulated in gallbladder cancer and inhibits cell growth through apoptosis induction by mitochondrial dysfunction. PLoS One. 2014; 9:1-10.

44. Miettinen M, Mccue PA, Sarlomo-rikala M, Biernat W, Czapiewski P, Kopczynski J, et al. Sox10 - a marker for not only Schwannian and melanocytic neoplasms but also Myoepithelial cell tumors of soft tissue. Am J Surg Pathol. 2015;39:826-35.

45. Tong X, Li L, Li X, Heng L, Zhong L, Su X, et al. SOX10, a novel HMG-boxcontaining tumor suppressor, inhibits growth and metastasis of digestive cancers by suppressing the wnt/ $\beta$-catenin pathway. Oncotarget. 2014;5: 10571-83.

46. Zhong W, Qin G, Dai Q, Han Z, Chen S, Ling X, et al. SOXs in human prostate cancer: implication as progression and prognosis factors. BMC Cancer. 2012:12:248.

47. Dittmer $\mathrm{J}$. The role of the transcription factor Ets 1 in carcinoma. Semin Cancer Biol. 2015:35:20-38.

\section{Publisher's Note}

Springer Nature remains neutral with regard to jurisdictional claims in published maps and institutional affiliations.

Ready to submit your research? Choose BMC and benefit from:

- fast, convenient online submission

- thorough peer review by experienced researchers in your field

- rapid publication on acceptance

- support for research data, including large and complex data types

- gold Open Access which fosters wider collaboration and increased citations

- maximum visibility for your research: over $100 \mathrm{M}$ website views per year

At $\mathrm{BMC}$, research is always in progress.

Learn more biomedcentral.com/submissions 